\title{
MANAGING A CLUSTER OF CRYPTOSPORIDIOSIS ASSOCIATED WITH A PUBLIC SWIMMING POOL
}

\section{Jennifer Paterson and Ian Goldthorpe \\ Sydney West Centre for Population Health}

Between 10 May and 20 June 2005, Sydney West Centre for Population Health (SWCPH) was notified of 29 cases of cryptosporidiosis. SWCPH staff interviewed these cases about their potential sources of exposure to infection and found that 11 (38 per cent) swam at the same indoor heated public pool. The onset of illness in these 11 cases ranged from late April to early June. Nine of these cases were children, six of whom were aged three years and under. Six of the eleven cases also reported other potential sources of infection, including contact with relatives who had diarrhoeal illness, contact with pets, and attendance at a childcare centre or play group.

The pool was used almost exclusively for learn-to-swim classes, which included non-toilet-trained infants. These infants are required to wear swimmers with waterproof tight fitting pants over them when they enter the pool. The pool operator reported using liquid stabilised chlorine dioxide in combination with super-chlorination on a regular basis in accordance with current NSW guidelines (see: http:// internal.health.nsw.gov.au/public-health/ehb/general/pools/ cryptopools.pdf).

Council staff checked the pool's testing logbook fortnightly, and had found no obvious problems in the management of the pool in the weeks before this cluster of cryptosporidiosis was identified.

In response to the outbreak, SWCPH staff contacted the pool manager and recommended that the pool be superchlorinated with a 'shock' dose of liquid stabilised chlorine dioxide on the evening of 24 May and with liquid sodium hypochlorite on the following evening. Liquid stabilised chlorine dioxide, which has recently become available, can 'clean' a pool in only two to four hours, whereas sodium hypochlorite, the traditional treatment, takes around 14 hours. Council staff re-tested the pool on 25 and 26 May and found that it met the requirements for an indoor heated swimming pool prescribed by the NSW guidelines.

However, following the identification of further cases, SWCPH staff arranged for the pool's water to be tested for Cryptosporidium oocysts on 17 June. The sample tested positive (636 oocysts in 107 litres). In response the pool was super-chlorinated on 17 June and again on 20 June. SWCPH staff also recommended that the pool management implement additional strategies to prevent contamination with Cryptosporidium. These included improved procedures for asking parents about diarrhoeal illness in themselves and their children and increasing the period of exclusion for individuals with diarrhoea from one week to two. No further cases of cryptosporidiosis have been associated with this pool. 줌 